
ОКОЛОЩИТОВИДНЫХ ЖЕЛЕЗ. ПИЛОТНОЕ ИССЛЕДОВАНИЕ

(с) Н.Г. Мокрышева ${ }^{1}$, И.А. Воронкова ${ }^{1,2 *}$, Ю.А. Крупинова ${ }^{1 *}$, М.Б. Долгушин ${ }^{3}$, Л.Е. Гуревич², А.А. Оджарова ${ }^{3}$, С.Н. Кузнецов ${ }^{1}$, И.В. Крюкова

1 ФГБУ «Национальный медицинский исследовательский центр эндокринологии» Минздрава России, Москва, Россия; 2 ГБУЗ МО «Московский областной научно-исследовательский клинический институт им. М.Ф. Владимирского», Москва, Россия;

${ }^{3}$ ФГБУ «Национальный медицинский исследовательский центр онкологии им. Н.Н. Блохина» Минздрава России, Москва, Россия

Обоснование. Первичный гиперпаратиреоз (ПГПТ) - широко распространенное эндокринное заболевание, характеризующееся избыточной продукцией паратиреоидного гормона (ПТГ) вследствие гиперплазии околощитовидных желез (ОЩЖ) или их опухолевого поражения (аденома или рак ОЩЖ, 80\% и 1-5\% случаев соответственно). Гиперэкспрессию холинкиназы-альфа (XКа) описывают в новообразованиях различной локализации, но данные о ее экспрессии в ОЩЖ отсутствуют.

Цель. Изучить характер экспрессии ХКа в новообразованиях ОЩЖ и ее связь с клиническими, лабораторными и визуализационными характеристиками (уровень накопления ${ }^{18} \mathrm{~F}$-фторхолина при позитронно-эмиссионной томографии, совмещенной с компьютерной томографией (ПЭТ/КТ)).

Методы. Материалом для исследования послужили образцы ткани образований ОЩЖ от 10 пациентов в возрасте 34-70 лет (Ме=61,5 [48;66]), с лабораторно подтвержденным диагнозом ПГПТ. Иммуногистохимическое исследование (ИГХ) проведено на материале от 2 пациентов с гиперплазией главных клеток, от 5 - с аденомой ОЩЖ, от 1 - с атипической аденомой, 1 - с карциномой ОЩЖ, в 1 случае исследовали метастаз рака ОЩЖ в лимфатический узел шеи. Результаты. Экспрессия ХКа отмечена во всех типах клеток ОЩЖ (главные клетки: активные (аГК) и неактивные (наГК) формы, переходные формы между главными клетками и оксифильными (оГК); оксифильные клетки (ОК)), но наиболее интенсивная экспрессия была зафиксирована в аГК. Экспрессия ХКа наблюдалась в новообразованиях ОЩЖ различной степени злокачественности. В самой многочисленной группе образований ОЩЖ с благоприятным прогнозом (11 образцов от 7 пациентов) не было получено статистически значимой корреляции (р>0,05) между интенсивностью экспрессии ХКа, экспрессией ПТГ и индексом пролиферативной активности Кі-67, уровнем накопления радиофармпрепарата при ПЭТ/KT с ${ }^{18} \mathrm{~F}-$ фторхолином (SUVmax) и лабораторными данными (ПTГ, Сa, Сa++).

Заключение. В большинстве случаев в клетках ОЩЖ выявлена умеренная и интенсивная экспрессия ХКа. Небольшое количество исследований не позволяет нам выявить взаимосвязь между интенсивностью экспрессии ХКа и злокачественным потенциалом образования ОЩЖ.

КЛЮЧЕВЫЕ СЛОВА: околощитовидные железы; холинкиназа-альфа; первичный гиперпаратиреоз; атипическая аденома околощитовидных желез; кариинома околощитовидных желез; позитронно-эмиссионная томография, совмещенная с компьютерной томографией $\mathrm{C}^{18} \mathrm{~F}$-фрторхолином.

\title{
EVALUATION OF THE METABOLISM PROPERTIES OF CHOLINE KINASE ALPHA IN NEOPLASMS OF THE PARATHYROID GLANDS. A PILOT STUDY
}

(c) Natalia G. Mokrysheva', lya A. Voronkova ${ }^{1 *}$, Julia A. Krupinova ${ }^{*}$, Mikhail B. Dolgushin³, Larisa E. Gurevch², Akgul A. Odzharova ${ }^{3}$, Sergei N. Kuznecov ${ }^{1}$, Irina V. Kryukova ${ }^{2}$

'Endocrinology Research Centre, Moscow, Russia;

${ }^{2}$ M.F. Vladimirskiy Moscow Regional Research and Clinical Institute, Moscow, Russia;

${ }^{3}$ «N.N. Blokhin National Medical Research Center of Oncology» of the Ministry of Health of the Russian Federation, Moscow, Russia

BACKGROUND: Primary hyperparathyroidism (PHPT) is a widespread endocrine disease characterized by excessive production of parathyroid hormone (PTH) due to parathyroid gland hyperplasia (PGH) or tumor lesions (adenoma or cancer of the parathyroid gland (PG) in $80 \%$ and $1-5 \%$ of cases respectively). Choline kinase a-alpha (XKa) overexpression is described in tumors of different localization, but there is no data on its expression in PG tumors. AIMS: To study the character of XKa expression in PG neoplasms and its relationship with clinical, laboratory, and visualization characteristics (positron emission tomography combined with computed tomography (PET/CT) with ${ }^{18} \mathrm{~F}-\mathrm{fluorocholine}$ $\left.\left({ }^{18} \mathrm{~F}-\mathrm{FC}\right)\right)$.

MATERIALS AND METHODS: The material for the study was based on tissue samples from 10 patients of 34-70 years old $(\mathrm{Me}=61.5 ;[48 ; 66])$, with a laboratory-confirmed diagnosis of PHT. An immunohistochemical study (IHC) was carried out on materials from 2 patients with hyperplasia of the main cells, from 5 patients with adenoma of PG, from 1 patient 
with atypical adenoma and 1 with carcinoma of PG; in 1 case the metastasis of cancer of the neck with lymph node was examined.

RESULTS: The expression of XKa is spotted in all types of PG cells (chief cells: active and inactive forms), transitional forms between the chief cells and oxyphil; oxyphil cells, but it was most intense in active chief cells. The expression of XKa was observed in neoplasms of PG of various degrees of malignancy. In the most numerous group of PG formations with a favorable prognosis (11 samples from 7 patients), no statistically significant correlation ( $p>0.05$ ) was obtained between the intensity expression of the $\mathrm{XKa}$, of the PTH and the proliferative activity index $\mathrm{Ki}-67$, the level of radiopharmaceutical accumulation in PET/CT with ${ }^{18} \mathrm{~F}-\mathrm{FC}$ (SUVmax) and laboratory data (PTH, Ca, $\mathrm{Ca}++$ ).

CONCLUSIONS: In the majority of investigated cases, moderate and intensive expression of the XKa was detected in PG cells. A small amount of studied cases does not allow us to identify the connection between the intensity of XKa expression and the malignant potential for the formation of PG.

KEYWORDS: parathyroid gland; choline kinase alpha; primary hyperparathyroidism; atypical parathyroid adenoma; parathyroid carcinoma; ${ }^{18} \mathrm{~F}$-Fluorocholine PET/CT.

\section{ОБОСНОВАНИЕ}

Первичный гиперпаратиреоз (ПГПТ) - широко распространенное эндокринное заболевание, характеризующееся избыточной продукцией паратиреоидного гормона (ПТГ), а также верхненормальным или повышенным уровнем кальция в крови вследствие гиперплазии или опухолевого поражения околощитовидных желез (ОЩЖ). Причиной развития ПГПТ в большинстве случаев является аденома ОЩЖ (около 80\%), реже - первичная гиперплазия, рак ОЩЖ диагностируется лишь у 1-5\% пациентов [1-2]. Учитывая неуклонный рост заболеваемости ПГПТ, можно прогнозировать, что и выявляемость злокачественных новообразований ОЩЖ будет расти. По данным американского регистра SEER (Surveillance, Epidemiology, and End Results), в США за 16 лет наблюдения (с 1988 по 2003 гг.) был зафиксирован рост заболеваемости раком ОЩЖ (с 1988 по 1991 гг. - 3,58 на 10 млн населения; с 2000 по 2003 гг. - 5,73 на 10 млн населения) [3]. Эпидемиологические данные указывают на необходимость поиска новых маркеров прогноза заболевания и потенциальных терапевтических мишеней.

Холинкиназа (ХК), открытая в 1953 г. Wittenberg и Kornberg [4], является первым ферментом в цикле Кеннеди [5] биосинтеза фосфатидилхолина (ФХ). ФХ - главный липид мембран эукариотических клеток, необходимый для обеспечения их структурной стабильности и способности к пролиферации [6]. ХK локализуется в цитоплазме клетки и катализирует реакцию трансформации холина в фосфохолин (ФХо) с использованием молекулы АТФ и $\mathrm{Mg}^{2+}$ в качестве кофактора. ФХо под действием фермента цитидиндифосфат-фосфохолинцитидилтрансферазы далее фосфорилируется до цитидиндифосфатхолина (ЦДФ-холин), затем в другие промежуточные соединения перед включением в фосфолипиды клеточной мембраны в виде ФХ [7-8].

У млекопитающих существует 3 изоформы ХK (XKa1, ХКа2 и ХК(), кодируемые двумя разными генами - СНКА и СНКВ. Данные гены у людей расположены в хромосомах 11q13.2 и 22q13.33 соответственно [7-9]. Оба гомодимера ХКа1 и ХКа2 обладают как холин- , так и этаноламин-киназной активностью. Гомодимер ХК $\beta$ преимущественно обладает этаноламин-киназной активностью, а гетеродимер ХКа-ХКß имеет промежуточную специфичность [7-8].

Кроме своей основной роли в метаболизме липидов клеток, ХК, предположительно, участвует в регуляции клеточной пролиферации и в канцерогенезе.
Аргументом в пользу существенной роли ХК в канцерогенезе является повышение ФХо во время трансформации клеток, опосредованной онкогеном RAS [8-10]. Вероятно, повышенная активность ХК в злокачественных опухолях является результатом гиперэкспрессии ХКа. В свою очередь, это может привести к более высокой доле димеров ХКа-ХКа и, соответственно, к более высокой активности XK, чем у гетеродимеров ХКа-ХK $\beta$ или гомодимеров ХK $\beta-X K \beta$ [7]. Существует гипотеза о влиянии ХКа на пролиферацию и трансформацию клеток путем регулирования смены фаз клеточного цикла G1-S и сигналов к апоптозу [11]. Гиперэкспрессия ХКа зафиксирована в опухолях молочной железы, легкого, толстой кишки, мочевого пузыря и предстательной железы человека[11-14].

Выраженная экспрессия ХКа в опухолевых клетках позволяет рассматривать ее как возможную мишень для терапевтического воздействия [15]. Lacal и соавт. в 1997 г. впервые предложили использовать ингибиторы СНКа в качестве потенциальной противоопухолевой терапии [15]. На сегодняшний день существует несколько поколений ингибиторов XKa (MN58b, TCD-717) $[7,15,16]$.

Радиоизотопное мечение холина дает возможность использовать его в качестве ПЭТ-индикатора. Во время фосфорилирования изотопная метка остается внутри клетки, что позволяет визуализировать ее метаболизм. В 2012 г. при проведении позитронно-эмиссионной томографии, совмещенной с компьютерной томографией (ПЭТ/КТ) с ${ }^{11} \mathrm{C}$-холином, у больного раком предстательной железы была случайно выявлена опухоль ОЩЖ, а впоследствии диагностирован ПгПТ. Такая же находка у пациента с аналогичным диагнозом продемонстрирована

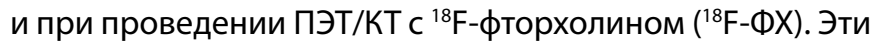
случаи показали возможность использования меченого холина для визуализации патологически измененных ОЩж [17-20]. В то же время оценка выраженности экспрессии ХКа с помощью иммуногистохимического (ИГХ) метода зарекомендовала себя при анализе образцов тканей опухолей молочной железы, легких и предстательной железы [21-22]. Ряд исследователей связывают степень и характер экспрессии ХКа с прогнозом заболевания $[12,14,16]$. Большинство из них склонны расценивать гиперэкспрессию ХКа как неблагоприятный прогностический фактор [12, 14, 23]. В настоящее время отсутствуют сведения об особенностях экспрессии ХКа в новообразованиях ОЩЖ. 
Нам представляется важным сбор данных об уровне накопления радиофармпрепарата (РФП) при ПЭТ/КТ c ${ }^{18} \mathrm{~F}-Ф X$ (SUVmax) в ОЩЖ и степени экспрессии XКа в тканях удаленных образований.

\section{ЦЕЛЬ}

Изучить характер и интенсивность экспрессии ХКа в новообразованиях ОЩЖ, выявить взаимосвязь между полученными характеристиками с клиническими, лабораторными данными (уровень ПТГ, $\mathrm{Ca}, \mathrm{Ca}++$ ) и уровнем накопления РФП при ПЭТ/КТ с ${ }^{18} \mathrm{~F}-Ф Х$.

\section{МЕТОДЫ}

\section{Дизайн исследования}

В обсервационное одномоментное неконтролируемое многоцентровое пилотное исследование включены 10 пациентов в возрасте от 37 до 70 лет. Хирургическое лечение ПГПТ в условиях ФГБУ «НМИЦ эндокринологии» Минздрава России проведено 9 пациентам и 1 - в ГБУз МО МОНИКИ им. М.Ф. Владимирского. ПЭТ/КТ с ${ }^{18} \mathrm{~F}-Ф Х$ на предоперационном этапе выполнено 8 больным в ФГБУ «НМИЦ онкологии им. Н.Н. Блохина» Минздрава России.

На срезах с парафиновых блоков проводили ИГХ-исследование с антителами к ПТГ, маркеру пролиферации Кi-67 и ХКа.

Интенсивность и характер экспрессии ХКа оценивались в гиперплазированных ОЩЖ и в новообразованиях ОЩЖ разной степени злокачественности. При смешанном клеточном составе образований особенности экспрессии ХКа оценивались отдельно в каждой клеточной популяции.

\section{Критерии соответствия}

В исследование вошли пациенты старше 18 лет с подтвержденным диагнозом ПГПТ, которым выполнено радикальное хирургическое лечение, что было подтверждено результатами лабораторного обследования (нормализация уровня ПТГ и $\mathrm{Ca}, \mathrm{Ca}++$ в послеоперационном периоде). Морфологическая диагностика новообразований ОЩЖ проводилась в соответствии с критериями классификации опухолей эндокринных органов (BO3, 2017).

\section{Условия проведения}

Оценка гистологических препаратов и ИГХ-реакции выполнена двумя патологами независимо с последующим обсуждением результатов и вынесением коллегиального заключения.

В исследование вошли пациенты, наблюдавшиеся и оперированные в ФГБУ «НМИЦ эндокринологии» Минздрава России и в ГБУЗ МО «МОНИКИ им. М.Ф. Владимирского». ПЭТ/КТ проводилось в ФГБУ «НМИЦ онкологии им. Н.Н. Блохина» Минздрава России.

Продолжительность исследования

Исследование проводилось с 2017 по 2018 гг.

\section{Основной исход исследования}

Основным результатом исследования считалось подтверждение наличия или отсутствия экспрессии ХКа в ткани новообразования ОЩЖ.

\section{Дополнительные исходы исследования}

В ходе исследования также были оценены следующие параметры: анализ связи характера экспрессии ХКа в новообразованиях ОЩЖ с клиническими, лабораторными и визуализационными характеристиками (уровень накопления ${ }^{18} \mathrm{~F}-Ф Х$ при ПЭТ/КТ).

\section{Анализ в подгруппах}

Для анализа полученных результатов мы выделили 3 группы: I - образования ОЩЖ с благоприятным прогнозом (10 образцов от 7 пациентов): аденомы (5 пациентов), гиперплазии ОЩЖ (2 случая; суммарно 5 ОЩЖ), II - новообразование с неопределенным потенциалом злокачественности (1 атипическая аденома) и III - злокачественные образования, включавшая 2 пациентов (1 случай - рак ОЩЖ и 1 случай - метастаз карциномы ОЩЖ в лимфатический узел шеи) (табл. 1). Сопоставление полученных результатов между группами не производилось ввиду слишком маленькой выборки. В группе I был проведен корреляционный анализ между лабораторными, визуализационными и морфологическими показателями. В случаях, когда у 1 пациента в патологический процесс было вовлечено несколько желез, лабораторные показатели дублировались для тех ОЩЖ, которые имели уровень SUV max более 1.

\section{Методы регистрации исходов}

Визуализацию образований ОЩЖ с помощью

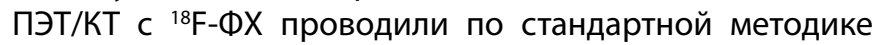
через 40 минут после внутривенного введения РФП (с предварительным соблюдением бесхолиновой диеты в течение 48 ч, натощак). Исследования выполняли на ПЭТ-камерах фирмы Siemens (Biograph mCT40).

Для измерения поглощения статичных изображений использован стандартизированный уровень накопления по максимальным значениям (SUV max (the standardized uptake value)), определяемый как отношение радиоактивности пикселя к введенной радиоактивности, нормализованной по весу.

ИГХ-исследование проведено с использованием первичных антител (AT) для ПтГ (клон MRQ-31; RTU; Cell Marque, США) и Кi-67 (клон МIB-1; разведение 1:100; Dako, Великобритания) по протоколам в соответствии с рекомендациями производителей. Для ХКа протокол отличался от рекомендованного и был подобран исходя из качества («чистоты») и выраженности ИГХ-реакции на образцах с показателем SUVmax выше 1 (polyclonal; разведение 1:10; Sigma-Aldrich, St. Louis, USA или Novus Biologicals, LLC, USA; температурное восстановление антигена в растворе со слабощелочной реакцией $\mathrm{pH}$, инкубация с первичными антителами в течение 2 ч). Для визуализации использована безбиотиновая DAB-система детекции.

Оценка экспрессии ПТГ и ХКа производилась полуколичественным методом по степени интенсивности цитоплазматического окрашивания: 0 «-» - отсутствие реакции, 1 «+»- слабая, 2 «+»- умеренная, 3 «+» - выраженная, 4 «+» - высокоинтенсивная. Также мы использовали $\mathrm{H}$-score для интерпретации результатов ИГХ-реакции с антитезами к ХКа, рассчитывая значения по формуле $(1 \times[\%$ клеток 1 «+»]) + $(2 \times$ [\% клеток 2 «+»]) $+(3 \times[\%$ клеток $3 \ll+»])+(4 \times[\%$ клеток 4 «+»]) [24]. Таким 
образом, при оценке ИГХ-реакции по шкале H-score уровень экспрессии находился в диапазоне 0 до 400.

Определение индекса Ki-67 рассчитано по стандартной методике, как среднее количество меченных этим маркером ядер, которое оценивается в процентах на 100 подсчитанных ядер опухолевых клеток, при учете 1000 клеток в репрезентативных полях зрения при большом увеличении (×400).

\section{Этическая экспертиза}

Все пациенты подписывали добровольное информированное согласие на использование биологического материала в научных целях и на обработку персональных данных.

\section{Статистический анализ}

Принципы расчета размера выборки

Объем выборки предварительно не рассчитывался.

Методы статистического анализа данных

Расчеты проводились при помощи пакета программы Statistica 12.

Описание количественных данных приведено в виде минимального и максимального значений, медианы (Me), 25 и 75 перцентилей. Взаимосвязь между получен- ными данными оценена с помощью коэффициента корреляции Спирмена $\left(r_{s}\right)$. Статистически значимыми считались результаты при $p<0,05$.

\section{РЕЗУЛЬТАТЫ}

Объекты (участники) исследования

В исследование вошли 10 пациентов в возрасте от 34 до 70 лет (Ме 61,5 [48;66]). Все пациенты имели лабораторно подтвержденный диагноз ПГПТ.

\section{Основные результаты исследования}

\section{Лабораторные данные}

Уровни ПТГ и Са были измерены 10 пациентам, уровень Са++ определяли 9 больным. В I группе значения ПТГ определялись в диапазоне от 215,4 до 1983 пг/мл (Ме 1298 [279,3;1682]), Са - от 2,84 до 4,09 ммоль/л (Ме 3,18 [2,99;3,24]), Са++ - от 1,36 до 1,91 (Ме 1,44; [1,36;1,62]) ммоль/л. Во ІІ когорте уровень ПТГ составил 2500 пг/мл, Са - 4,1 ммоль/л, измерение уровня Са++ не проводилось. В III группе у пациента с раком ОЩЖ и у больного с метастазом карциномы ОЩЖ уровень ПТГ составил 1049 и 291 пг/мл, Са - 2,59 и 3,14 ммоль/л, Са++ - 1,18 и 1,49 ммоль/л соответственно (табл. 1).

Таблица 1. Результаты морфологического и ИГХ-исследования и параметры ПЭТ/КТ с ${ }^{18} \mathrm{~F}-Ф Х$

\begin{tabular}{|c|c|c|c|c|c|c|c|c|c|}
\hline \multirow[b]{2}{*}{ 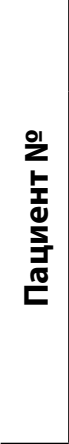 } & \multicolumn{4}{|c|}{ Морфологические данные } & \multicolumn{4}{|c|}{ Результаты ИГХ-исследования } & \multirow{2}{*}{ 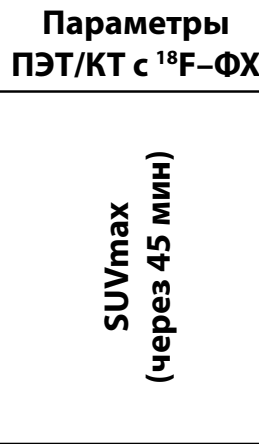 } \\
\hline & 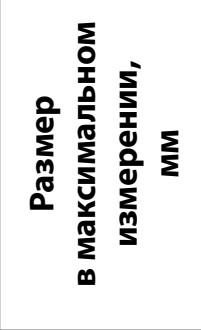 & $\begin{array}{l}\text { L } \\
\text { ப̇ } \\
\text { D }\end{array}$ & 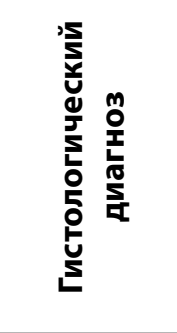 & 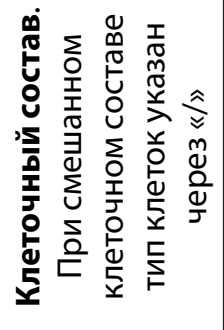 & 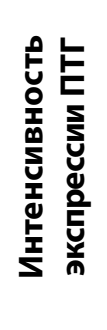 & 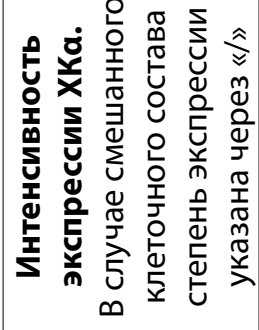 & 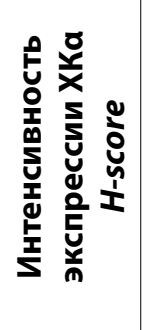 & 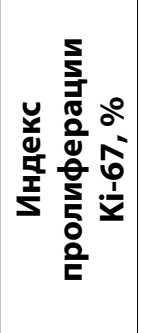 & \\
\hline \multirow{3}{*}{1} & $\begin{array}{l}\text { А.40 (правая } \\
\text { нижняя) }\end{array}$ & 74 & ГклГ & $\mathrm{a} \Gamma \mathrm{K}^{*}$ & 2 & 3 & 300 & 1 & 17,09 \\
\hline & $\begin{array}{l}\text { Б.7 (левая } \\
\text { верхняя) }\end{array}$ & НД & ГклГ & аГК*/наГК & 3 & 4 и $2 * / 2$ & 280 & 2 & 2,74 \\
\hline & $\begin{array}{l}\text { В.5 (левая } \\
\text { нижняя) }\end{array}$ & НД & ГклГ & $\mathrm{a} \Gamma \mathrm{K}^{*}$ & 4 & 4 & 400 & 1 & 1 \\
\hline \multirow{2}{*}{2} & $\begin{array}{l}\text { А.35 (левая } \\
\text { верхняя) }\end{array}$ & НД & ГклГ & $\mathrm{a} \Gamma \mathrm{K}^{*} / \mathrm{O}$ & 2 & $3 * / 2$ & 300 & 3 & 6,0 \\
\hline & $\begin{array}{l}\text { Б.55 (нижняя } \\
\text { правая) }\end{array}$ & 89 & ГклГ & $\mathrm{a} \Gamma \mathrm{K}^{*} / \mathrm{O}$ & 2 & $3 * / 2$ & 255 & 4 & 10,6 \\
\hline 3 & 45 & 94 & A & $\mathrm{a} \Gamma \mathrm{K}^{*}$ & 4 & 2 & 200 & 3 & 8,6 \\
\hline 4 & 40 & 63 & A & аГК*/наГК & 3 & $1 * / 3$ & 140 & 3 & 8,11 \\
\hline 5 & 10 & 10 & A & аГК*/наГК & 3 & $3 * / 2$ & 270 & 1 & 2,11 \\
\hline 6 & 55 & 50 & A & $\mathrm{a} \Gamma \mathrm{K}^{*}$ & 3 & 3 & 300 & 4 & 10,12 \\
\hline 7 & 45 & 57 & $A$ & аГК*/наГК/О & 3 & 4 и $3 * / 3 / 3$ & 310 & 4 & 9,0 \\
\hline 8 & 45 & 45 & AA & O*/oГK* & 3 & $2 * / 3 *$ & 250 & 22 & 4,39 \\
\hline 9 & 30 & НД & $\mathrm{P}$ & аГК*/наГК & НД & $4,3^{*}, 2 / 2$ & 280 & 11 & НД \\
\hline 10 & НД & НД & Mts Р в л/у & $\mathrm{a} \Gamma K^{*}$ & НД & 2 & 200 & 33 & НД \\
\hline
\end{tabular}

A - аденома; ГклГ - главноклеточная гиперплазия; АA - атипическая аденома; P - рак; Мts P в л/у - метастаз рака в лимфатический узел; аГК - активные главные клетки; наГК - неактивные главные клетки; ОК - оксифильные клетки; оГК - переходная форма между ГК и ОК;

* - преобладающий тип клеток; ** - типы клеток представлены в равных пропорциях; НД - нет данных. 

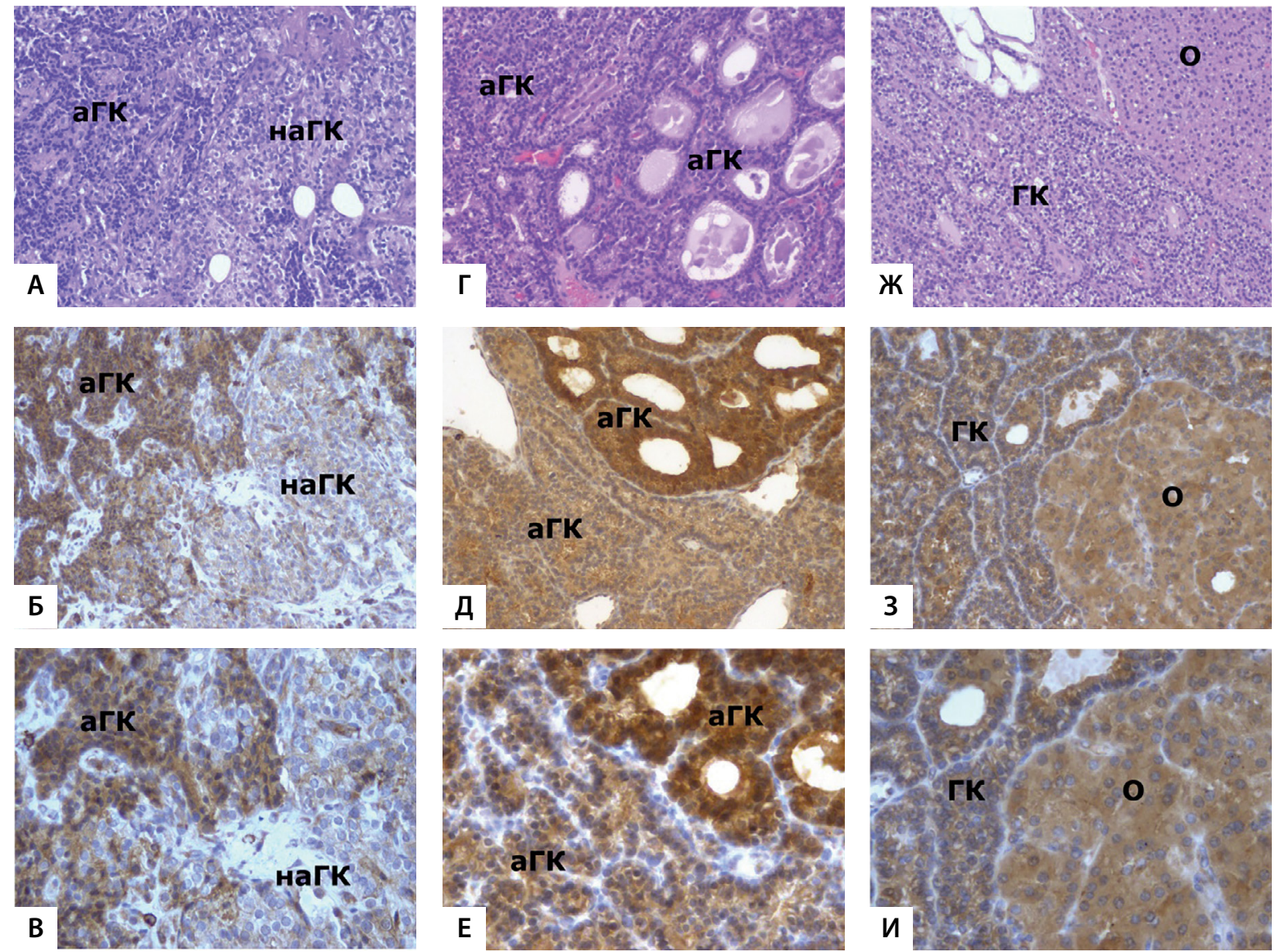

Рисунок 1. Экспрессия холинкиназы альфа (ХКа) в различных клеточных пулах в одном тканевом образце.

A-B - случай № 1Б; А - аГК и наГК (Г-Э ×200); Б и В - экспрессия ХКа интенсивная (4 «+») в аГК и умеренная (2 «+») в наГК (×200 и ×400 соответственно); Г-E - случай №7; Г - аГК, формирующие различные структуры (фолликулярные и трабекулярные); Д и Е - различная степень экспрессии ХКа в 4 «+» и 3 «+» В аГК (×200 и ×400 соответственно); Ж-И - случай № 7; Ж-О и ГК в разных фазах секреторного цикла (аГК и наГК) (Г-Эх100); 3 и И - выраженная (3+) экспрессия ХКа в обоих типах клеток ( $\times 200$ и $\times 400$ соответственно).

\section{Результаты ПЭт/КT с ${ }^{18} \mathrm{~F}-\Phi \mathrm{X}$}

ПЭТ/КТ с ${ }^{18} \mathrm{~F}-Ф Х$ проведена 8 пациентам, 2 пациентам С карциномами данное исследование не выполнялось. В 2 случаях из 8 у 1 больного визуализировалось 2 образования ОЩЖ. SUVmax был зафиксирован в диапазоне 2,11-17,09 (Ме 8,355 [4,39;10,12]) (см. табл. 1). В 1 случае интраоперационно было заподозрено, что в патологический процесс дополнительно вовлечена ОЩЖ, в которой не определялось повышенного накопления РФП, что, вероятно, связано с малым размером (5 мм), в данном случае уровень SUVmax был принят за 1 (см. табл. 1).

\section{Морфологическое исследование}

У 2 пациентов было удалено более одной ОЩЖ. Таким образом, для морфологического исследования доступно 13 образцов ткани ОЩЖ (см. табл. 1). Материал исследован от 2 пациентов с морфологическим диагнозом «главноклеточная гиперплазия» (в одном случае в патологический процесс было вовлечено 3, во втором - 2 ОЩЖ), 5 случаев с диагнозом «аденома ОЩЖ», 1 пациент с атипической аденомой, 1 пациент с карциномой ОЩЖ, в 1 случае исследовали метастаз рака ОЩЖ в лимфатический узел шеи (см. табл. 1).

\section{Результаты иммуногистохимического исследования}

ИГХ-исследование экспрессии ХКа

При проведении ИГХ-реакции мы наблюдали в клетках преимущественно цитоплазматическое окрашивание; очаговое мембранное окрашивание отмечено в 1 случае и в 2 случаях - мембранно-цитоплазмати-

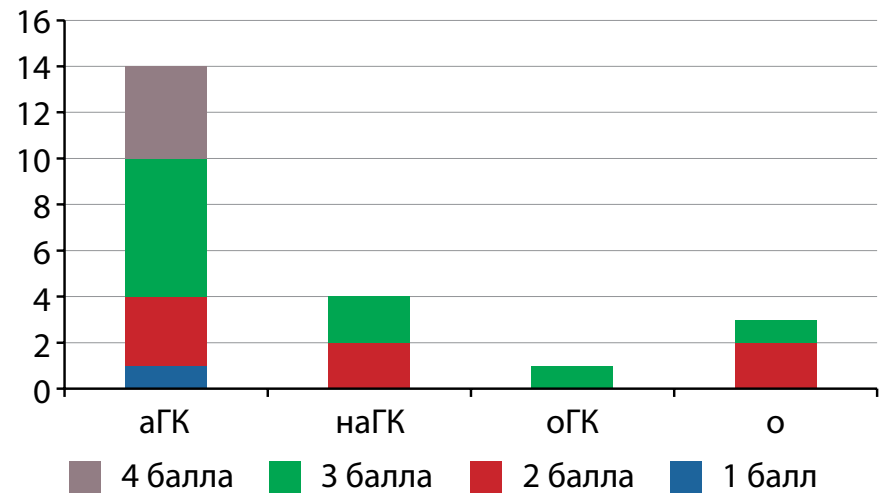

Рисунок 2. Распределение экспрессии холинкиназы альфа в баллах в зависимости от типа клеток образований ОЩЖ.

ческое, в 1 случае очагово отмечалось окрашивание по типу «dot like».

Анализируя ИГХ-реакцию с АТ к ХКа, мы отметили различия в интенсивности экспрессии данного маркера и в локализации иммунореактивного материала в клетках, которая могла различаться как в пределах одного клеточного пула, так и в разных типах клеток (главные клетки: активные (аГК) и неактивные (наГК) формы, переходная форма между главными клетками и оксифильными (оГК); оксифильные (О) клетки).

Экспрессия ХКа была выявлена во всех типах клеток, но наиболее интенсивной она была в аГК (см. табл. 1, рис. 1 и 2). Экспрессия ХКа наблюдалась 
в новообразованиях ОЩЖ различной степени злокачественности (см. табл. 1, рис. 3 и 4).

ИГХ-исследование экспрессии ПТГ

ИГХ-анализ с АТ к ПТГ был проведен во всех образцах, кроме злокачественных образований ОЩЖ. Интенсивная экспрессия ПТГ наблюдалась в I и II группах (см. табл. 1).

Уровень экспрессии маркера пролиферации Кі-67

Значение индекса пролиферации Кі-67 в группе образований ОЩЖ с благоприятным прогнозом составило $1-4 \%$ (Ме 3\% [1;4]), в атипической аденоме он был равен

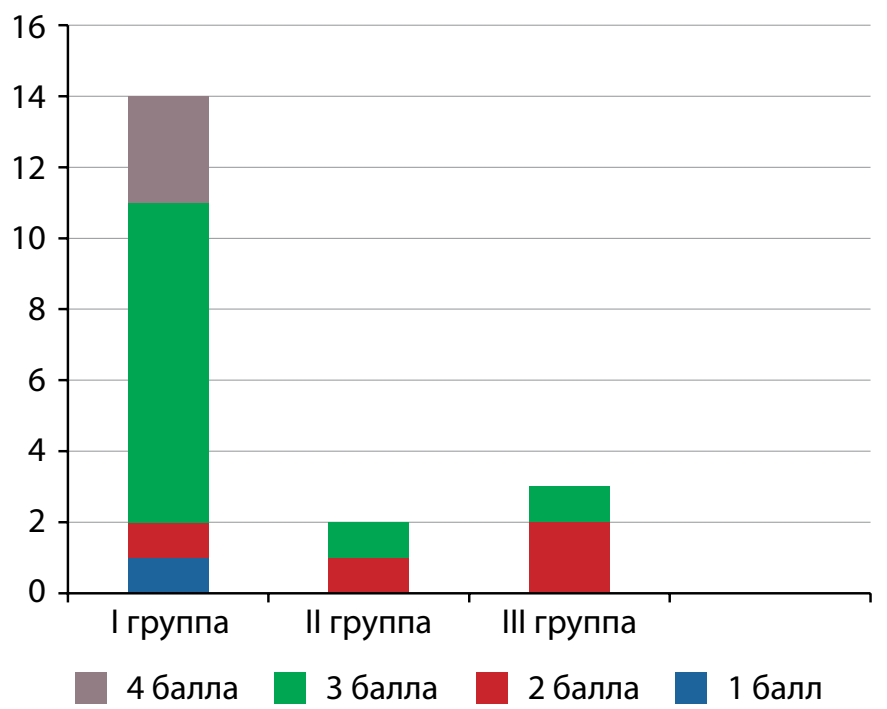

Рисунок 3. Распределение экспрессии холинкиназы альфа в баллах по преобладающей экспрессии (в баллах) в клетках В зависимости от степени злокачественности образований околощитовидных желез.

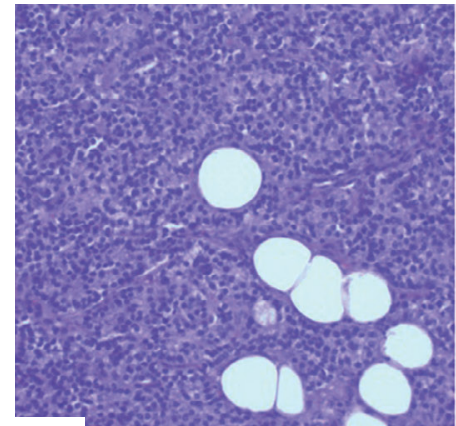

A
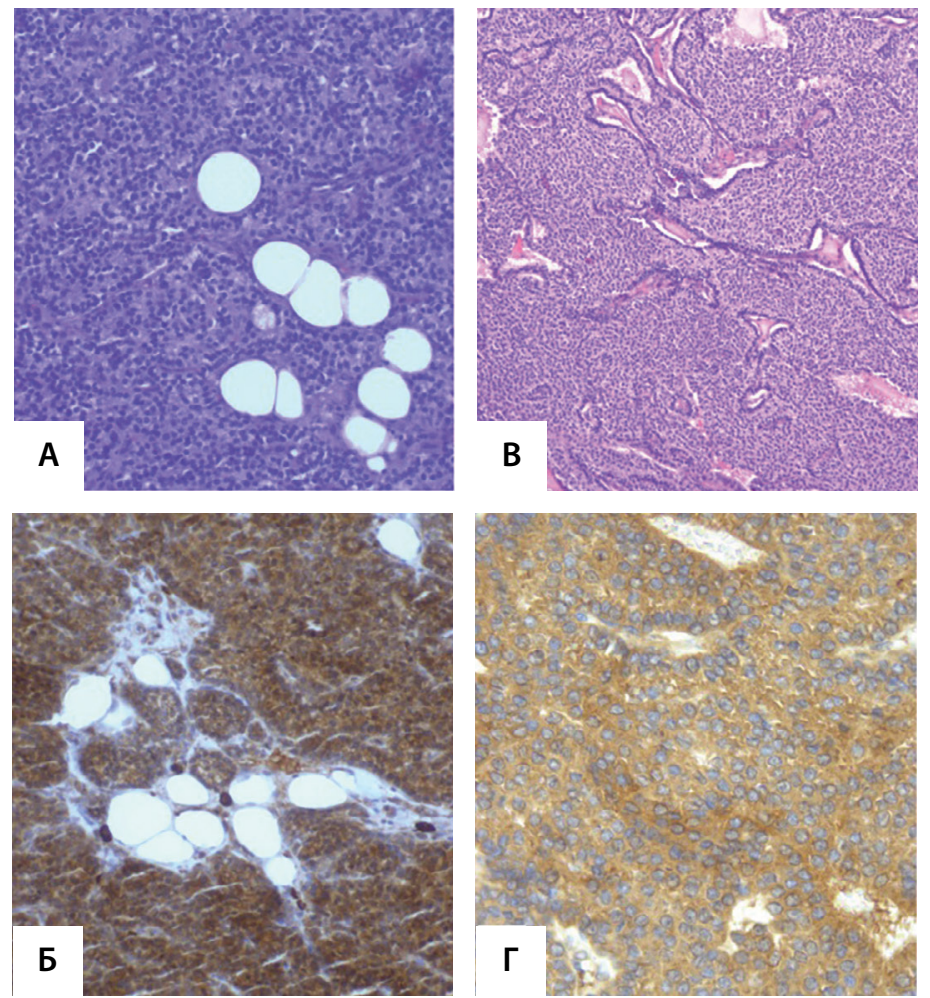

22\%, в карциноме - 11\%, а в метастазе рака ОЩЖ в лимфатический узел составил 33\% (см. табл. 1).

\section{Дополнительные результаты исследования}

Мы оценили наличие взаимосвязи между лабораторными (уровень ПТГ, Са и Са++, уровнем накопления РФП при ПЭТ/KT (SUVmax)) и ИГХ (экспрессия ХКа, ПТГ, индекс Кі-67) показателями в I, наиболее многочисленной (7 наблюдений) группе пациентов. При этом не получено статистически значимой ( $>>0,05)$ корреляции между экспрессией ХКа как по преобладающему типу клеток, так и по клеточному очагу с наибольшей интенсивностью экспрессии ПТГ и при расчете экспрессии по шкале H-score, с индексом Ki-67 ( $p=0,665 ; p=0,736 ; p=0,739$ соответственно), с экспрессией ПТГ клетками опухоли $(\mathrm{p}=0,315 ; \mathrm{p}=0,672 ; \mathrm{p}=0,755$ соответственно), с уровнем накопления РФП при ПЭТ/КТ $c{ }^{18} \mathrm{~F}-$ ФX (SUVmax) ( $p=0,850 ; p=0,296 ; p=0,774$ соответственно) и лабораторными данными: ПТГ ( $p=0,777 ; p=0,718$; $p=0,394)$, Са ( $p=0,837 ; p=0,899 ; p=0,394$ соответственно), Ca++ ( $p=0,566 ; p=0,717 ; p=0,985$ соответственно).

\section{Дополнительное наблюдение, выявленное в ходе} исследования

Анализируя ИГХ-реакцию с АТ к ХКа, мы обратили внимание на интенсивную экспрессию (4«+») данного маркера в отдельных клетках (не паратиреоцитах) в периваскулярной зоне (рис. 5), в большинстве исследуемых образцов. Для того чтобы раскрыть природу данного явления, необходимы дальнейшие исследования.

\section{Нежелательные явления}

Нежелательные явления не зарегистрированы.
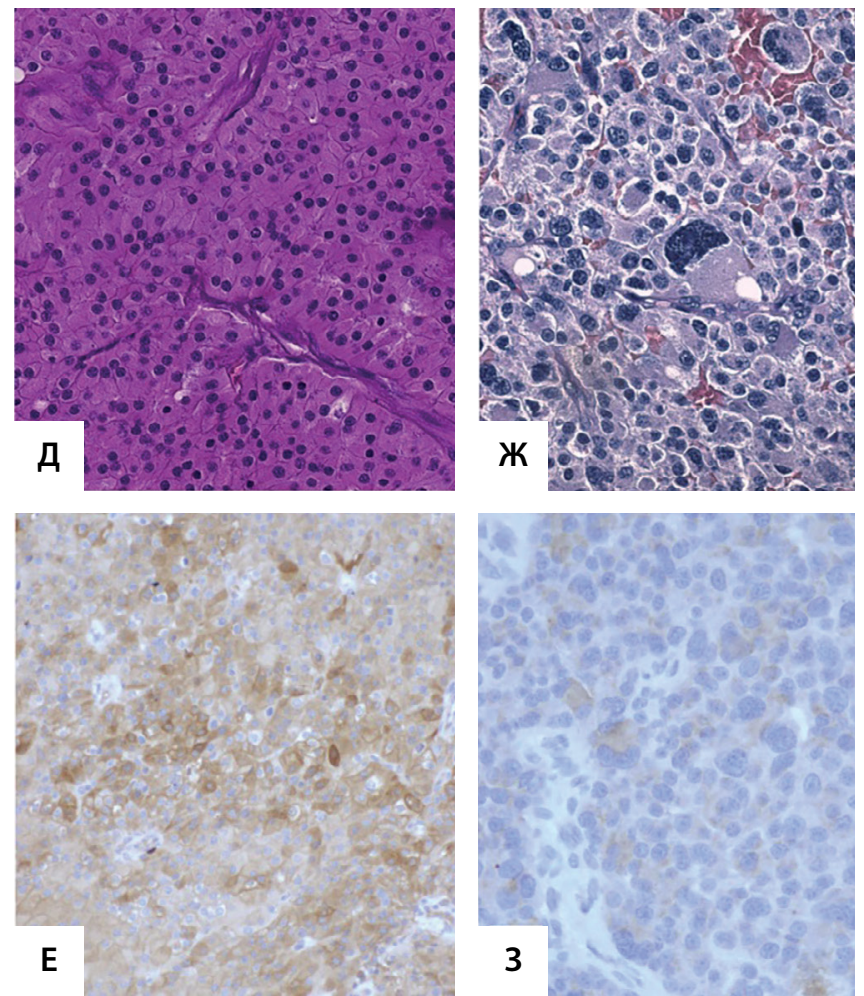

Рисунок 4. Экспрессия холинкиназы альфа (ХКа) в различных по степени злокачественности новообразованиях околощитовидных желез.

А и Б - главноклеточная гиперплазия ОЩЖ (случай №1B), клетки которой обладают интенсивной (4+) экспрессией ХКа (А. Г-Э ×200; Б. ИГХ × 200); В и Г - аденома ОЩЖ из главных клеток (случай №6), обладающая выраженной (3+) экспрессией ХКа (В. Г-Э ×200; Г. ИГХ ×200); Д и Е - карцинома ОЩЖ (случай №9) с умеренной (2+) и выраженной (3+) экспрессией ХКа (Д. Г-Э ×300; Е. ИГХ ×200); Ж и 3 - метастаз рака ОЩЖ в лимфатический узел (случай №10), умеренно (2+) экспрессирующий ХКа (Ж. Г-Э×400; 3. ИГХ ×400). 


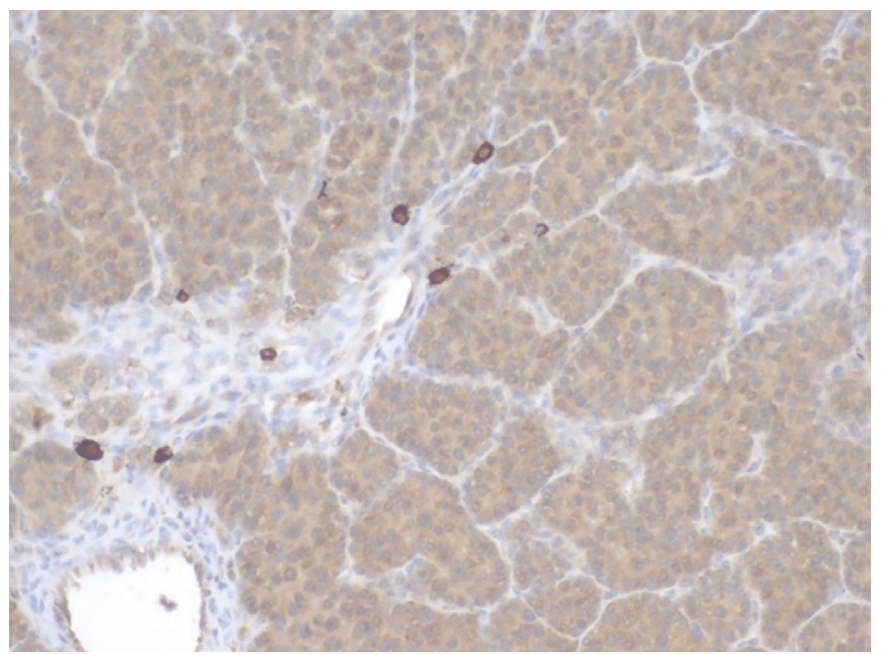

Рисунок 5. Выраженная (3+) экспрессия холинкиназы альфа в ткани аденомы околощитовидных желез и очень интенсивная (4+) в отдельных клетках периваскулярной зоны $(\times 200)$.

\section{ОБСУЖДЕНИЕ}

Резюме основного результата исследования

Экспрессия ХКа отмечена во всех типах клеток ОЩЖ (аГК, наГК, оГК; О), с максимальной интенсивностью (4 «+») в аГК. Экспрессия ХКа наблюдалась в новообразованиях ОЩЖ различной степени злокачественности.

\section{Обсуждение основного результата исследования}

При проведении ИГХ-исследования мы выявили преимущественно цитоплазматическую реакцию с антителами к ХКа, в 3 случаях - мембранную. Однако ряд исследователей отмечали и учитывали не только цитоплазматическое окрашивание, но и ядерное [16-25]. Contractor К. и соавт. при ИГХ-исследовании указали наличие ядерного окрашивания в 1 из 20 раков предстательной железы и в 1 случае простатической интраэпителиальной неоплазии (ПИН). Mazarico J.M. и соавт. сообщают о ядерном окрашивании в 43\% (всего 96 пациентов) протоковых аденокарцином поджелудочной железы, что в приведенной работе было связано с более благоприятным прогнозом [16]. Феномен наличия ядерной экспрессии ХКа еще не до конца изучен. Существует несколько гипотез: 1) наличие ядерной экспрессии обусловлено способностью ХКа фосфорилироваться и перемещаться в ядро вместе с другими белками [25]; 2) ХКа может транслоцироваться в ядро для синтеза эндонуклеарного фосфатидилхолина [12]. Мембранная экспрессия, по нашему мнению, может быть обусловлена перемещением ХКа к месту ее непосредственного функционального использования либо оттеснением этого фермента к периферии крупными внутриклеточными вакуолями и/или включениями. На сегодняшний день не решен вопрос о единой методике оценки экспрессии данного маркера. Так, в одних работах учитывалась только интенсивность экспрессии ХКа $[12,26]$, а в других принимали во внимание не только интенсивность, но и количество иммунопозитивных клеток $[14,16,23]$. При этом способы оценки количества и интенсивности экспрессии фермента у разных групп исследователей различались.
В данной работе выраженная экспрессия ХКа наблюдалась как в группе образований ОЩЖ с благоприятным прогнозом, так и в группе с неопределенным потенциалом злокачественности и в злокачественных новообразованиях. К сожалению, сопоставление полученных результатов по группам не производилось ввиду малой выборки. Большинство авторов отмечают, что в опухолях наблюдается более интенсивная экспрессия XКа, чем в тканях без патологических изменений $[12,16,25]$. Однако встречаются работы с противоположными результатами. В пилотном исследовании Kwee S.A. и соавт. в 5 холангиокарциномах была выявлена умеренная экспрессия ХКа в 2 балла (использовалась шкала от 0 до 3 баллов); в 3 случаях в неизмененной ткани печени экспрессия ХКа была сравнимой по интенсивности (2 балла), в двух других оценена в 1 балл [26]. Тем не менее наличие интенсивной экспрессии ХКа в доброкачественных образованиях, а также тот факт, что в ряде случаев при низком уровне SUVmax мы выявили интенсивную экспрессию ХКа, позволили нам рассмотреть ХКа как потенциальный маркер функциональной активности ОЩЖ, участвующий в механизмах секреции птг клетками ОЩЖ. Так, паратиреоциты при активном синтезе ПтГ увеличивают свой объем, а выход везикул с гормоном и разрыв клеточной мембраны требуют быстрого и массивного ее восстановления [27]. Можно предположить, что увеличение поверхности клеточной стенки приводит к интенсивному захвату холина и, следовательно, к более интенсивному уровню накопления ${ }^{18} \mathrm{~F}-Ф Х$ при проведении ПЭТ/КТ. Для синтеза ФХ из холина расходуется запас ХКа, а, следовательно, снижается уровень ее экспрессии в ткани. Таким образом, в гормонально более активных клетках уровень экспрессии ХКа будет ниже при более высокой степени накопления ${ }^{18} \mathrm{~F}-Ф Х$ при ПЭТ/КТ. Гипотезу об экспрессии ХКа и функциональной активности клеток можно косвенно подтвердить результатами нашего исследования, поскольку максимальная экспрессия данного маркера (4 «+») определялась в активных клетках. При этом аГК с экспрессией ХК в 4 балла преобладали лишь в 1 случае и, возможно, характеризовали начало секреторного цикла, в то время как неактивные клетки накапливают вещества, необходимые для следующей фазы. В то же время в одной аденоме, где остаток ткани ОЩЖ был широко предоставлен, экспрессия ХКа была несколько более интенсивной в неизмененном ободке (рис. 6). Схожие различия в экспрессии между тканью аденомы и ободком нормальной ОЩЖ выявлены в работе Haglund и соавт., где изучалась экспрессия ПТГ, которая чаще была более интенсивной в нормальной ткани ОЩЖ, нежели в клетках аденомы [28]. Различная степень экспрессии ХКа также может быть обусловлена ультраструктурными особенностями клеток ОЩЖ.

Нельзя оставить без внимания и тот факт, что микроокружение опухоли (например, гипоксия или рН межклеточной среды) могут влиять на метаболизм холина [7, 29]. Характер экспрессии может быть связан не только с типом секреции, но и с гистогенезом клеточных линий. В пользу подобной гипотезы говорит наблюдение Mazarico J.M. и соавт., которые проанализировали экспрессию ХКа во внеопухолевой ткани поджелудочной железы [16]. Более выраженная реакция наблюдалась в островковых клетках, чем в протоковых 

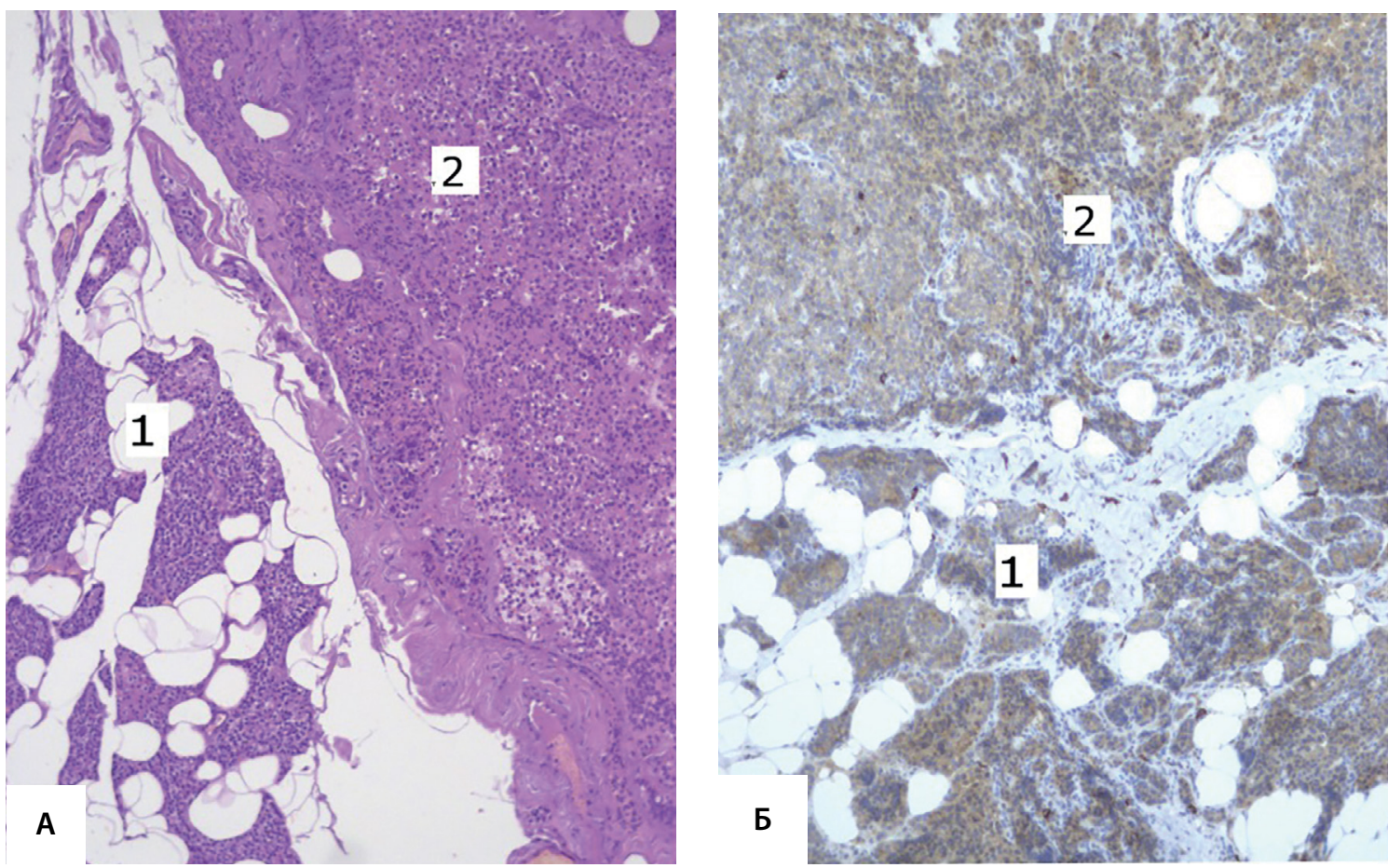

Рисунок 6. Аденома околощитовидных желез (случай №5)

А. Остаток ткани ОЩЖ (1) прилежит к ткани аденомы (2) преимущественно из главных клеток в разных фазах секреторного цикла, преобладают аГК (×50). Б. Выраженная (3 «+») экспрессия ХКа в ткани остатка ОЩЖ и выраженная (3 «+») и умеренная (2 «+») в ткани аденомы $(\times 100)$

и ацинарных [16]. Таким образом, предположение о ХКа как о возможном маркере гормональной активности клеток нуждается в дальнейших исследованиях на более обширном материале. Последующие работы, направленные на накопление данных о характере и особенностях экспрессии ХКа в образованиях ОЩЖ и взаимосвязи их с уровнем накопления РФП (SUVmax) при ПЭТ/KT c ${ }^{18} \mathrm{~F}-Ф X$, возможно, позволят использовать эти показатели в качестве дифференциально диагностических маркеров, факторов прогноза течения болезни и эффективности ответа на возможную терапию ингибиторами XКа

\section{Обсуждение дополнительных результатов}

\section{исследования}

В нашем исследовании не было выявлено статистически значимой корреляции между экспрессией ХКа и другими исследованными маркерами (экспрессия ПТГ и значение индекса пролиферации Кі-67). Отсутствие связи между экспрессией ХКа и значением индекса Кі-67 также было зафиксировано в работах Contractor К. и соавт. [25]. Результаты, полученные Aboagye и Bhujwalla [13], противоречат гипотезе о том, что высокий уровень ФХо является маркером повышенной пролиферативной активности клеток. В своей работе авторы, используя нормальные эпителиальные клетки ткани молочной железы и простаты с помощью гормона роста, ускорили их клеточное деление до уровня, характерного для раковых клеток, и получили уровень ФХо ниже, чем это наблюдалось в раковых клетках $[7,13]$. Аналогично этому Challapalli A. и соавт., исследовав 100 образцов рака предстательной железы и не обнаружив взаимосвязи между степенью экспрессии ХК и индексом пролиферации Кі-67, высказали предположение, что ХК может быть независимым маркером рака предстательной железы, который не отражает пролиферативную активность его клеток [12].
Мы не получили статистически значимой корреляции между экспрессией ХКа и уровнем накопления РФП при ПЭТ/КТ с ${ }^{18} \mathrm{~F}-Ф X$ (SUVMax) в группе доброкачественных образований ОЩЖ. В то время как Contractor K. и соавт. продемонстрировали прямую сильную зависимость между SUVmax [11C]choline ПЭТ/КТ и интенсивностью ИГХ-реакции с антителами к ХКа в карциномах предстательной железы ( $r=0,63$; $\mathrm{p}=0,0004)$ [25]. Данное противоречие может объясняться как малой выборкой в нашем исследовании, так и различным потенциалом злокачественности исследуемых образований (в нашей когорте ПЭТ/КТ с ${ }^{18} \mathrm{~F}-Ф Х$ не было выполнено ни одному пациенту со злокачественным новообразованием).

В данной группе мы также не выявили статистически значимой ( $>>0,05)$ корреляции между экспрессией XКа и лабораторными показателями (ПТГ, Са Са++).

\section{Ограничения исследования}

Ограничением данного исследования являются ма-

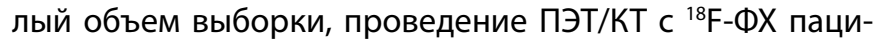
ентам только с доброкачественными образованиями ОЩЖ, отсутствие в выборке тканей нормальных ОЩЖ и с подавленной функцией, недостаток данных о возможных положительных внутренних контролях, субъективность оценки результатов ИГХ-исследования, отсутствие единого стандартизованного протокола проведения ИГХ-реакции с антителами к ХКа и метода регистрации результатов.

\section{ЗАКЛЮЧЕНИЕ}

В проведенном исследовании мы показали, что даже при отсутствии накопления ${ }^{18} \mathrm{~F}-Ф Х$ при ПЭТ/КТ клетки ОЩЖ экспрессируют ХКа. В большинстве случаев была 
выявлена умеренная и интенсивная экспрессия ХКа в ткани ОЩЖ. Небольшое количество исследований и малая выборка не позволяют нам выявить зависимость между особенностями экспрессии ХКа и злокачественным потенциалом образований. В ходе эксперимента в наиболее многочисленной группе образований ОЩж с благоприятным прогнозом (11 образцов от 7 пациентов) не выявлено статистически значимой корреляции между интенсивностью экспрессии ХКа в клетках образований, лабораторными данными и уровнем накопления ${ }^{18} \mathrm{~F}-Ф Х$ при ПЭТ/КТ. Однако на основе полученных данных можно высказать гипотезу о том, что экспрессия ХКа может характеризовать гормональную активность клеток ОЩЖ.

Принимая во внимание отсутствие достаточного количества данных о визуализации ОЩЖ с помощью ПЭТ/КТ с ${ }^{18} \mathrm{~F}$-ФХ и его прогностическом значении, о наличии взаимосвязи показателя SUVmax с интенсивностью экспрессии ХКа и потенциалом злокачественности в образованиях различной локализации и гистогенетического происхождения, дальнейшие исследования в этом направлении представляются перспективными и актуальными. Необходимо продолжить эти исследования с возможностью получить более обширные и углубленные данные, накопить опыт о значении и взаимозависимости всех изученных показателей в ткани ОЩЖ при различных патологических процессах.

\section{ДОПОЛНИТЕЛЬНАЯ ИНФОРМАЦИЯ}

Источник финансирования. Публикация настоящей работы поддержана государственным заданием «Наследственные опухолевые синдромы и множественные эндокринные неоплазии: персонализация диагностики и лечения, прогнозирование рисков, идентификация ядерных семей». Номер государственного учета НИОКТР АААА-А18-118051590057-2

Конфликт интересов. Авторы декларируют отсутствие явных и потенциальных конфликтов интересов, связанных с публикацией настоящей статьи.

Участие авторов. Мокрышева Н.Г. - разработка и руководство исследованием, концепция статьи, анализ литературных данных, написание и редактирование текста; Воронкова И.А. - анализ литературных данных, проведение морфологического исследования, подготовка микрофотографий, написание и редактирование текста; Крупинова Ю.А. - отбор и ведение пациентов, анализ литературных данных, написание и редактирование текста; Долгушин М.Б. - выполнение ПЭТ/КТ с ${ }^{18} \mathrm{~F}-Ф Х$, анализ литературных данных, написание и редактирование текста; Гуревич Л.Е. - анализ литературных данных, проведение морфологического исследования, отбор микрофотографий, редактирование текста.; Оджарова А.А. - выпол-

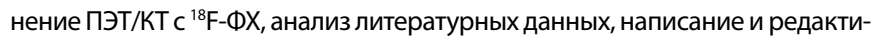
рование текста; Кузнецов С.Н. - выполнение хирургического лечения, анализ литературных данных, редактирование текста; Крюкова И.В. - ведение пациента, анализ литературных данных, редактирование текста.

Все авторы внесли значимый вклад в проведение исследования и подготовку статьи, прочли и одобрили финальную версию статьи перед публикацией.

\section{СПИСОК ЛИТЕРАТУРЫ | REFERENCES}

1. Favia G, Lumachi F, Polistina F, D’Amico DF. Parathyroid Carcinoma: Sixteen New Cases and Suggestions for Correct Management. World J Surg. 1998;22(12):1225-1230. doi: https://doi.org/10.1007/s002689900549

2. Shane E. Parathyroid Carcinoma. J Clin Endocrinol Metab. 2001;86(2):485-493. doi: https://doi.org/10.1210/jcem.86.2.7207

3. Lee PK, Jarosek SL, Virnig BA, et al. Trends in the incidence and treatment of parathyroid cancer in the United States. Cancer. 2007;109(9):1736-1741. doi: https://doi.org/10.1002/cncr.22599

4. Wittenberg J, Kornberg A. Choline phosphokinase. J Biol Chem. 1953:202(1):431-444. PMID: 13061469

5. Gibellini F, Smith TK. The Kennedy pathway-De novo synthesis of phosphatidylethanolamine and phosphatidylcholine. IUBMB Life. 2010;62(6):414-428. doi: https://doi.org/10.1002/iub.337

6. Zeisel SH. Dietary Choline: Biochemistry, Physiology, and Pharmacology. Daya S, ed. Annu Rev Nutr. 1981;1 (1):95-121. doi: https://doi.org/10.1146/annurev.nu.01.070181.000523

7. Glunde K, Bhujwalla ZM, Ronen SM. Choline metabolism in malignant transformation. Daya S, ed. Nat Rev Cancer. 2011;11(12):835-848. doi: https://doi.org/10.1038/nrc3162

8. Патент РФ на изобретение № 2509809/ 20.03.2014 Бюл. № 8 . Лакаль Акаль Санхуан Хуан Карлос, Рамирес де Молина Ана, Галлего Ортега Давид. Способы лечения и диагностики рака. [Patent RUS №2509809/ 20.03.2014. Byul. №8. Lakal' Akal' Sankhuan Khuan Karlos, Ramires de Molina Ana, Gallego Ortega David. Sposoby lecheniya i diagnostiki raka (In Russ)]. Доступно по http://www.fips.ru/cdfi/fips.dll/ru?ty=29\&docid=2509809 Ссылка активна на 17.06.2019.

9. HGNC [Internet]. HUGO Gene Nomenclature Committee. The resource for approved human gene nomenclature. [Last updated: 2019-06-16]. Available from: https://www.genenames.org/

10. Ramírez de Molina A, Rodríguez-González A, Penalva V, et al. Inhibition of ChoK Is an Efficient Antitumor Strategy for HarveyKirsten-, and N-ras-Transformed Cells. Biochem Biophys Res Commun. 2001;285(4):873-879. doi: https://doi.org/10.1006/bbrc.2001.5250

11. Ramírez de Molina A, Gallego-Ortega D, Sarmentero-Estrada J, et al. Choline kinase as a link connecting phospholipid metabolism and cell cycle regulation: Implications in cancer therapy. Int J Biochem Cell Biol. 2008:40(9):1753-1763.

doi: https://doi.org/10.1016/j.biocel.2008.01.013

12. Challapalli A, Trousil S, Hazell S, et al. Exploiting altered patterns of choline kinase-alpha expression on human prostate tissue to prognosticate prostate cancer. J Clin Pathol. 2015;68(9):703-709. doi: https://doi.org/10.1136/jclinpath-2015-202859

13. Aboagye EO, Bhujwalla ZM. Malignant transformation alters membrane choline phospholipid metabolism of human mammary epithelial cells. Cancer Res. 1999:59(1):80-84. PMID: 9892190

14. Hu L, Wang R-Y, Cai J, et al. Overexpression of CHKA contributes to tumor progression and metastasis and predicts poor prognosis in colorectal carcinoma. Oncotarget. 2016;7(41):703-709. doi: https://doi.org/10.18632/oncotarget.11433

15. Lacal JC. Choline kinase: a novel target for antitumor drugs. IDrugs. 2001;4(4):419-426. PMID: 16015482

16. Mazarico JM, Sanchez-Arevalo Lobo VJ, Favicchio R, et al. Choline Kinase Alpha (CHK) as a Therapeutic Target in Pancreatic Ductal Adenocarcinoma: Expression, Predictive Value, and Sensitivity to Inhibitors. Mol Cancer Ther. 2016;15(2):323-333. doi: https://doi.org/10.1158/1535-7163.MCT-15-0214

17. Quak E, LheureuX S, Reznik Y, et al. F18-Choline, a Novel PET Tracer for Parathyroid Adenoma? J Clin Endocrinol Metab. 2013;98(8):3111-3112. doi: https://doi.org/10.1210/jc.2013-2084

18. Hodolic M, Huchet V, Balogova S, et al. Incidental uptake of $18 \mathrm{~F}$-fluorocholine $(\mathrm{FCH})$ in the head or in the neck of patients with prostate cancer. Radiol Oncol. 2014;48(3):228-234. doi: https://doi.org/10.2478/raon-2013-0075

19. Cazaentre T, Clivaz F, Triponez F. False-Positive Result in 18F-Fluorocholine PET/CT Due to Incidental and Ectopic Parathyroid Hyperplasia. Clin Nucl Med. 2014;36(6):e328-e330. doi: https://doi.org/10.1097/RLU.0b013e3182a77b62

20. Мокрышева Н.Г., Крупинова Ю.А., Долгушин М.Б., и др. Позитронная эмиссионная томография, совмещенная с компьютерной томографией с 18F- фторхолином в топической диагностике опухолей околощитовидных желез и вторичных изменений костной ткани при гиперпаратиреоидной остеодистрофии. Два клинических наблюдения // Проблемь эндокринологии. — 2018. - Т.64. — №5. - С.299-306. 
[Mokrysheva NG, Krupinova JA, Dolgushin MB, et al. Positron emission tomography in combination with computed tomography with $18 \mathrm{~F}-$ fluorocholine in the topical diagnosis of parathyroid tumors and secondary changes in bone tissue associated with hyperparathyroid osteodystrophy: two case studies. Problems of Endocrinology. 2018;64(5):299-306. (In Russ).] doi: http://doi.org/10.14341/probl9548

21. Gallego-Ortega D, Ramirez De Molina A, Gutierrez R, et al. Generation and characterization of monoclonal antibodies against choline kinase $a$ and their potential use as diagnostic tools in cancer. Int J Oncol. 2006;109(9):1736-1741. doi: https://doi.org/10.3892/ijo.29.2.335

22. Ramírez de Molina A, Gutiérrez R, Ramos MA, et al. Increased choline kinase activity in human breast carcinomas: clinical evidence for a potential novel antitumor strategy. Oncogene. 2002;21(27):43174322. doi: https://doi.org/10.1038/sj.onc.1205556

23. Zhang $L, C$ Chen $P$, Yang $S$, et al. CHKA mediates the poor prognosis of lung adenocarcinoma and acts as a prognostic indicator. Oncol Lett. 2016;12(3):1849-1853. doi: https://doi.org/10.3892/ol.2016.4810

24. Silva-Figueroa A, Villalobos P, Williams MD, et al. Characterizing parathyroid carcinomas and atypical neoplasms based on the expression of programmed death-ligand 1 expression and the presence of tumor-infiltrating lymphocytes and macrophages. Surgery. 2018;164(5):960-964. doi: https://doi.org/10.1016/j.surg.2018.06.013

25. Contractor K, Challapalli A, Barwick T, et al. Use of [11C]Choline PET-CT as a Noninvasive Method for Detecting Pelvic Lymph Node Status from Prostate Cancer and Relationship with Choline Kinase Expression. Clin Cancer Res. 2011;17(24):7673-7683. doi: https://doi.org/10.1158/1078-0432.CCR-11-2048

26. Kwee SA, Okimoto GS, Chan OT, et al. Metabolic characteristics distinguishing intrahepatic cholangiocarcinoma: a negative pilot study of (18)F-fluorocholine PET/CT clarified by transcriptomic analysis. Am J Nucl Med Mol Imaging. 2016;6(1):73-83.

27. Mihai R. The Calcium Sensing Receptor: From Understanding Parathyroid Calcium Homeostasis to Bone Metastases. Ann R Coll Surg Engl. 2008;90(4):271-277. doi: https://doi.org/10.1308/003588408X286044

28. Haglund F, Juhlin CC, Kiss NB, et al. Diffuse PTH expression in parathyroid tumors argues against important functional tumor subclones. Eur J Endocrinol. 2016;174(5):583-590. doi: https://doi.org/10.1530/EJE-15-1062

29. Gillies RJ, Raghunand N, Karczmar GS, Bhujwalla ZM. MRI of the tumor microenvironment. J Magn Reson Imaging. 2002;16(4):430-450. doi: https://doi.org/10.1002/jmri.10181

\section{ИНФОРМАЦИЯ ОБ АВТОРАХ [AUTHORS INFO]:}

*Воронкова Ия Александровна, к.м.н., н.с. [lya A. Voronkova, MD, PhD]; адрес: Россия, 117036, Москва, ул. Дм. Ульянова, д. 11 [address: 11 Dm. Ulyanova street, 117036 Moscow, Russia]; ORCID:http://orcid.org/0000-0001-6687-3240; eLibrary SPIN: 9685-1371; e-mail: iya-v@yandex.ru

* Крупинова Юлия Александровна, н.с. [Julia A. Krupinova, MD, researcher]; адрес: Россия, 117036, Москва, ул. Дм. Ульянова, д. 11 [address: 11 Dm. Ulyanova street, 117036 Moscow, Russia]; ORCID: http://orcid.org/0000-0001-7963-5022; eLibrary SPIN: 6279-8247; e-mail: j.krupinova@gmail.com

Мокрышева Наталья Георгиевна, д.м.н., профессор [Natalia G. Mokrysheva, MD, PhD, Professor]; ORCID: http://orcid.org/0000-0002-9717-9742; eLibrary SPIN: 5624-3875; e-mail: nm70@mail.ru Долгушин Михаил Борисович, д.м.н., профессор [Mikhail B. Dolgushin, MD, PhD, Professor]; ORCID: https://orcid.org/0000-0003-3930-5998; eLibrary SPIN-код: 6388-9644; e-mail: mdolgushin@mail.ru Гуревич Лариса Евсеевна, д.б.н., г.н.с., профессор [Larisa E. Gurevch, PhD in biology, Professor]; ORCID: orcid.org/0000-0002-9731-3649; eLibrary SPIN: 8615-0038; e-mail: larisgur@mail.ru

Оджарова Акгуль Атаевна, к.м.н. [Akgul A. Odzharova, MD, PhD, researcher]; ORCID: https://orcid.org/0000-0003-3576-6156; eLibrary SPIN: 1074-3862; e-mail: odzharova07@yandex.ru Кузнецов Сергей Николаевич, хирург, н.с. [Sergey N. Kuznetsov, MD, surgeon, researcher]; ORCID: https://orcid.org/0000-0001-8558-7725; eLibrary SPIN: 9870-2578; e-mail: kuznetsov_enc@mail.ru Крюкова Ирина Викторовна, К.м.н. [Irina V. Kryukova, MD, PhD]; ORCID:http://orcid.org/0000-0002-7876-5105; eLibrary SPIN: 7669-3010; e-mail: kiv200877@yandex.ru

*Авторы, ответственные за переписку.

\section{ЦИТИРОВАТЬ:}

Мокрышева Н.Г., Воронкова И.А., Крупинова Ю.А., Долгушин М.Б., Гуревич Л.Е., Оджарова А.А., Кузнецов С.Н., Крюкова И.В. Оценка особенностей метаболизма холинкиназы альфа в новообразованиях околощитовидных желез. Пилотное исследование // Ожирение и метаболизм. - 2019. - Т.16. — №3. - С.94-103. doi: https://doi.org/10.14341/omet10260

\section{TO CITE THIS ARTICLE:}

Mokrysheva NG, Voronkova IA, Krupinova JA, Dolgushin MB, Gurevch LE, Odzharova AA, Kuznecov SN, Kryukova IV. Evaluation of the metabolism properties of choline kinase alpha in neoplasms of the parathyroid glands. Pilot study. Obesity and metabolism. 2019;16(3):94-103. doi: https://doi.org/10.14341/omet10260 